\title{
PELIGRO AVIARIO: CARACTERIZACIÓN DE LA VEGETACIÓN Y DETERMINACIÓN DE LAS ESPECIES QUE SON ATRACTIVAS PARA LA FAUNA SILVESTRE EN LAS ÁREAS VERDES DEL AEROPUERTO INTERNACIONAL AUGUSTO C. SANDINO, MANAGUA, NICARAGUA
}

\author{
José Benito Quezada', Carlos Ruiz Fonseca², Miguel Garmendia Zapata', Luís Chavarría Ocón ${ }^{3}$, \\ Wesbter Lanuza Morán ${ }^{4}$ \\ ${ }^{1}$ Lic. Docente de la Facultad de Recursos Naturales y del Ambiente, FARENA/UNA \\ ${ }^{2}$ MSc. Docente de la Facultad de Ciencia Animal, FACA/UNA \\ ${ }^{3}$ Ingeniero Forestal, consultor independiente \\ ${ }^{4}$ Ingeniero en Recursos Naturales Renovables, consultor independiente
}

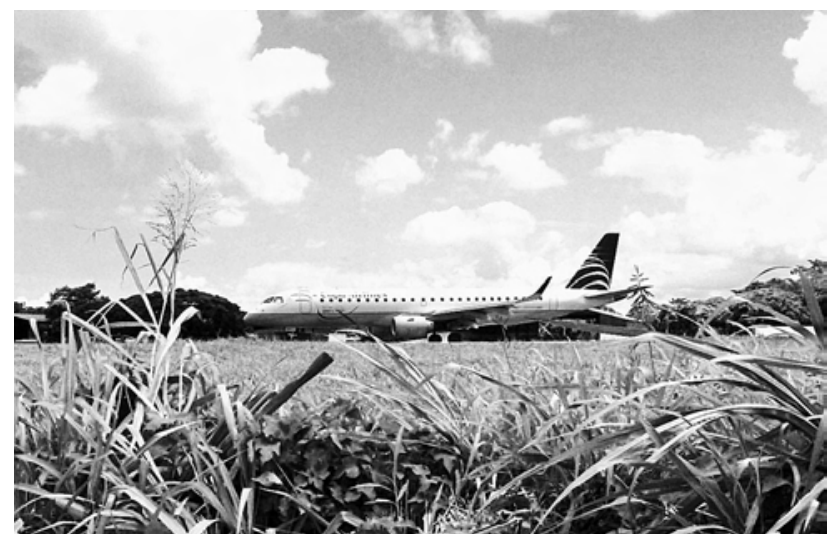

\section{RESUMEN}

Se evaluó la diversidad, estructura y fenología de la flora en las áreas verdes del Aeropuerto Internacional Augusto C. Sandino con el fin de determinar las especies que son atractivas para fauna silvestre de peligro para la aviación, sea por que les provee de alimento o de refugio y hábitat. Y por otro lado, brindar recomendaciones sobre especies con potencial para formar parte de las áreas verdes del aeropuerto. Para ello, se establecieron parcelas anidadas de $2 \times 2 \mathrm{~m}, 5 \mathrm{x}$ $5 \mathrm{~m}$ y $10 \times 10 \mathrm{~m}$ en las cuales se tomaron los datos en los estratos herbáceos, arbustivos y arbóreos respectivamente. Las parcelas se establecieron aleatoriamente en tres sitios dentro del aeropuerto: en el bosque seco al oeste del aeropuerto (BSO), en la Fuerza Aérea (FA) y en el área verde alrededor de la pista de aterrizaje (AVP). Dentro de las parcelas se contaron 117 árboles en todos los sitios, los cuales estaban agrupados en 11 especies, 10 géneros, 8 familias y 4 órdenes. Las especies arbóreas de mayor densidad fueron: Calycophyllum candidissimum (450 individuos/ ha), Albizia niopides (344), Azadirachta indica (289) y Senna siamea (261) y el sitio que presento mayor diversidad y densidad de árboles fue FA. Fueron contados 36 individuos de arbustos, pertenecientes a las especies Capsicum annum (1) y Lantana camara (35). En cuanto a especies herbáceas se contaron 11,845 individuos dentro de las unidades de muestreo, agrupados en 28 especies, 23 géneros, 14 familias y 13 órdenes, siendo el AVP el sitio que registró mayor diversidad y densidad de las mismas. Las especies herbáceas con mayores densidades fueron Cenchrus brownii (256,282.05

\section{ABSTRACT}

Diversity, structures and phenology of plants inside green areas of Augusto C. Sandino International Airport were evaluated. The purpose was to determine the species that are attractive to dangerous wild fauna, due to the fact that those species can provide food, refuge and habitats for animals. With this study, we expect recommend species with potential to be planted on the green areas of the airport. Nested plots of $2 \times 2 \mathrm{~m}, 5 \times 5 \mathrm{~m}$ and $10 \times 10 \mathrm{~m}$ were established to take the data of herbs, bushes and trees respectively. Plots were established randomly in three places inside the airport field area: the west dry forest of the airport (BSO), the air force (FA) and in the green area around the landing strip (AVP). Inside the plots, 117 trees were counted in all places, all of then included in 11 species, 10 genus, 8 families and 4 orders. The trees species with highest densities were: Calycophyllum candidissimum (450 individuals/ ha), Albizia niopides (344), Azadirachta indica (289) and Senna siamea (261). The place with the biggest diversity and density of trees was FA. Thirty six individuals of bushes were identified, one belonging to Capsicum annum and 35 to Lantana camara. Eleven thousand eight hundred forty five individuals of herbs were counted, derived from 28 species, 23 genus, 14 families and 13 orders. AVP got the highest diversity and density of herb. Herbs species with highest densities were: Cenchrus brownii (256,282.05 individuals/ha), Cynodon dactylon (141,538.46), Digitaria decumbens $(106,794.87)$, Bothriochloa pertusa $(51,282.05)$, Elyrtaria imbricada $(38,846.15)$ y Panicum maximum $(30,192 \cdot 31)$. Complete phenology was determined 
individuos/ha), Cynodon dactylon (141,538.46), Digitaria decumbens $(106,794.87)$, Bothriochloa pertusa $(51,282.05)$, Elytaria imbricada $(38,846.15)$ y Panicum maximum $(30,192.31)$. De las 41 especies vegetales, se determinó la fenología completa para 31, de estas, 6 (19.35\%) florecen y fructifican todo el año: Cordia dentata, Boerhavia erecta, Chamaesyce hyssopifolia, Eleucine indica, Melanthera nivea y Rhynchosia minima. Entre las especies arbóreas mayormente atractivas para la fauna silvestre se determinaron: Albizia niopoides, Manguifera indica, Spondias mombin, Cordia dentata, Guazuma ulmifolia y Calycophyllum candidissimum al ser considerados proveedores de alimentos y refugio a la fauna silvestre, por sus frutos, flores con abundante néctar, copas con poca obstrucción visual (alta densidad de hojas) y ramificaciones horizontales propias para el perchaje de las aves de alto tamaño. Dentro de la vegetación herbáceas se determinaron especies como Tridax procumbens y Tribulus terrestis formando densos tapetes de vegetación con flores atractivas para insectos en el período seco, los cuales a la vez eran focos de atracción para aves insectívoras como: Hirundus rustica (Golondrinas), Quiscalus mexicanus (Zanate), Molotrus aeneus (Tordos), Egretta thula (Garza blanca) que permanecen en las áreas verdes alrededor de la pista de aterrizaje y que peligran colisionar con los aviones. Por otra parte, las especies de la familia Poaceae fueron registradas como proveedoras de granos a las aves del grupo de las Columbidae (Palomas). Especies de gramíneas de gran porte como Panicum maximum, P. antidotale, Sorgum halapensis, además de poseer semillas grandes $(>0.3 \mathrm{~cm})$, formaban corredores por donde se desplazan mamífero medianos, reptiles y aves. Dentro del mismo áreas se determinaron dos especies (B. pertusa y $C$. dactylon) que por su baja estatura (menos de $0.3 \mathrm{~m}$ ), reproducción vegetativa y semillas muy pequeña o ausentes, son ideales para establecer en los alrededores de la pista de aterrizaje. Mediante un ensayo se evaluó su establecimiento, el cual resulto satisfactorio al competir y ganar espacio ante otras monocotiledones y dicotiledonesas, principalmente $C$. dactylon .

Palabras clave: alimento, hábitat, refugio, Poaceae to 31 species, 6 (19.35\%) got flowers and fruits all the year: Cordia dentata, Boerhavia erecta, Chamaesyce hyssopifolia, Eleucine indica, Melanthera nivea y Rhynchosia minima. The trees species as Albizia niopoides, Manguifera indica, Spondias mombin, Cordia dentata, Guazuma ulmifolia y Calycophyllum candidissimum were determined as attractive to wildlife, they supply them foods and refuge, because fruits, flowers with abundant nectar, leaves with less visual obstruction (high densities of leaves) and horizontal branches. Herbs species such as Tridax procumbens and Tribulus terrestis were determined forming dense mats of vegetation with attractive flowers to insects in the dry season, those insects at the same time, were focuses of attraction for insectivorous birds as: Hirundus rustica (Swallows), Quiscalus mexicanus (Zanate), Molotrus aeneus (Starling), Egretta thula (White Egretts) which remain in the green areas around the runway and were dangerous to strike with airplanes. Species of Poaceae family has been registered as supplying of grains to birds as Columbidae (Doves), very abundant around the runway. Large species of Poaceae as Panicum maximum, $P$. antidotale, Sorgum halapensis, produce big grains $(>0.3$ $\mathrm{cm})$ and formed corridors to medium large mammal, reptiles and others birds. Inside airport areas, two species (B. pertusa and $C$. dactylon) were determined with not very potential as attractiveness for wildlife, mainly to be small (less than 0.3 $\mathrm{m})$, vegetative reproduction and small grains or without them, excellent to plant on the surroundings the runway. An essay to establish both species was carried out, with satisfactory results; they won space against others monocotiledons and dicotiledons plants, especially $C$. dactylon.

Keywords: food, habitat, refuge, Poaceae
$\mathrm{E}$ 1 Aeropuerto Internacional Augusto C. Sandino (antes campo de aviación Xolotlán), ubicado en la parte norte-este la ciudad de Managua constituye una de las principales vías de comunicación de la Nicaragua con el mundo, siendo este el principal puerto aéreo de intercambio de mercadería, valores económicos y culturales, el cual data desde 1931 (EAAI, 2006). Este espacio internacional aeroportuario al igual que otros del mundo y sobre todo de países en desarrollo, presenta una serie de problemas de operación y de seguridad, en este último caso sobresalen los riesgos por problemas aviarios (el riesgo que las aves representan para las operaciones aeroportuarias por el peligro de choques entre aves y aeronaves se define como "Peligro Aviario") marcados fundamentalmente por las condiciones ambientales favorables que se presentan en la parte interna y periférica de las pistas de aterrizaje. Las especies de fauna silvestre son atraídas debido al alimento, agua o hábitat que el aeropuerto les proporcione, el tamaño de las poblaciones atraídas por el entorno aeroportuario varían considerablemente, dependiendo de varios factores, incluyendo actividades de uso de suelo dentro o cerca del aeropuerto (Cleary \& Dolbeer, 2005).

Desde un inicio la superficie de rodamiento de la pista de aterrizaje de Aeropuerto Internacional Augusto 
C. Sandino ha sido terreno inicialmente cubierto por grama y actualmente por una variada vegetación que sirve de albergue y foco de alimentación de aves, mamíferos medianos y de reptiles, que de forma directa o indirecta ponen en riesgo las operaciones aeroportuaria.

Los expertos en riesgo aviario internacionalmente señalan que el conocimiento del hábitat, sobre todo de la flora, es fundamental para la elaboración de futuros planes de manejo de los hábitats que atraen fauna de riesgo para los aeropuertos, es por ello que la Universidad Nacional Agraria (UNA) en conjunto con la Empresa Administradora del Aeropuerto Internacional (EAAI), a solicitud de estos últimos, se planteo la ejecución del presente trabajo, con la finalidad de determinar la diversidad de la vegetación existente dentro del área de la pista de aterrizaje y despegue de las aeronaves, donde se identifico la riqueza vegetal, su estructura, fenología, condiciones de hábitat, así como de alimento atractivo para la fauna silvestre y en base a ello brindar recomendaciones de manejo, que permitan manipular la vegetación a fin de reducir la presencia de fauna de riesgo para la aviación.

Además se planteo dentro del estudio la identificación y selección de especies poco atractivas para la fauna silvestre, las cuales se puedan estudiar y recomendar para esparcir dentro de las áreas verdes del aeropuerto y hacer un recambio de la vegetación, sustituyendo aquellas especies vegetales muy atractivas.

\section{MATERIALES Y MÉTODO}

Descripción general del área de estudio. El aeropuerto Internacional Augusto C. Sandino se encuentra ubicado aproximadamente a 11.5 kilómetros del centro de la ciudad de Managua en el sector oriental entre las coordenadas $12^{\circ} 08^{\prime} 30^{\prime \prime}$ latitud norte y $86^{\circ} 10^{\prime} 24^{\prime \prime}$ longitud oeste. Ocupa un predio de aproximadamente 354.34 hectáreas distribuidas en forma casi rectangular, con 3,600m de Este a Oeste y $800 \mathrm{~m}$ de Norte a Sur, elevación de 58.2msnm (EAAI, 2006). Las temperaturas se registra entre 22.6 y $32.9^{\circ} \mathrm{C}$, con precipitaciones entre 63.1 a $132.3 \mathrm{~mm}$ anuales y humedad relativa media del $74.2 \%$. Las velocidades del viento se registran entre 0.2 hasta $3.8 \mathrm{~m} / \mathrm{s}$. Presión atmosférica de 1004.1 Hpa y nubosidad de 2.0 octas.

Proceso metodológico. El proceso metodológico para llevar a cabo el cumplimiento de los objetivos está dividida en dos partes: 1 . Metodología para caracterizar de la vegetación en el área de estudio y 2 . Metodología para la selección y evaluación del establecimiento de especies de gramíneas poco atractivas para la fauna silvestre de peligro para la aviación. En el primer caso se establecieron unidades de muestreo que consistieron en parcelas anidadas de 2 × $2 \mathrm{~m}, 5 \times 5 \mathrm{~m}$ y $10 \times 10 \mathrm{~m}$ en donde se tomaron datos para evaluar las especies de plantas herbáceas, arbustivas y arbóreas respectivamente. Los conjuntos de parcelas se distribuyeron mediante un diseño de muestreo aleatorio estratificado, en donde el área de estudio se dividió en estratos o sitios y en cada sitio se establecieron las unidades de muestreo de una forma aleatoria. Dichos sitios se nombraron de la siguiente manera: la Fuerza Aérea (FA), el bosque seco al oeste del aeropuerto (BSO) y el área verde alrededor de la pista de aterrizaje (AVP) (Figura 1). El número total de parcelas establecidas fue de 78, 16 de $10 \times 10 \mathrm{~m}$ (estableciéndose nueve en el BSO, seis en la FA y una en el AVP), tres de 5 x 5m (dos en el AVP y una en FA) y 59 de 2 x 2 m ( 39 en AVP y 20 en BSO).

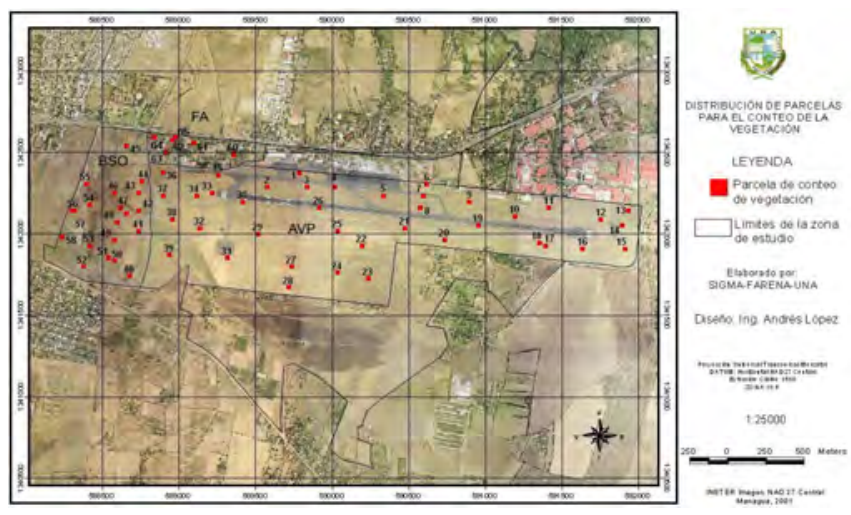

Figura 1. Ubicación de los conjuntos de parcelas para la evaluación de la composición florística en el área verde del Aeropuerto Augusto C. Sandino.

Las variables que se tomaban dentro de cada parcela fueron: número de especies, número de individuos, alturas de las plantas y fenología (floración y fructificación). Todos esos datos fueron útiles para calcular: abundancia, riqueza, densidad, diversidad de especies y para determinar las especies atractivas para la fauna silvestre. La diversidad se calculó mediante el uso del índice de diversidad biológica de Shannon-Wiener (Moreno, 2001), y se calcularon las especies esperadas mediante el estimador no paramétrico Chao2 (Moreno, 2001). Para todos los análisis antes mencionados se utilizaron los programas de computo: EstimateS (Colwell, 2004) y PAST (Hammer \& Harper, 2004).

Como medida de tendencia central se utilizó la media aritmética o promedio. El nivel de significancia empleado fue el de 0.05 . Las pruebas se movían en las dos colas. Ante de las pruebas de hipótesis a todos los datos se les aplicó una prueba de normalidad de 
Kolmogorov-Smirnov, en caso de que los datos no se ajustaran a la normalidad se decidió usar una prueba homóloga no paramétrica. En los análisis de frecuencia se utilizó la prueba de ji cuadrada $\left(\mathrm{X}^{2}\right)$ y como prueba de promedios ANOVA o T-student o sus homólogas no paramétricas Kruskal-Wallis o Mann-Whitney, según el caso. Las estadísticas se aplicaron mediante uso del programa de computo InStat (GraphPad Software, 2000).

Selección y evaluación de gramíneas para establecerse en las áreas verdes del aeropuerto. Se seleccionaron dos especies como potenciales para establecer en las áreas verdes del aeropuerto, dentro de los criterio de selección se encuentran su baja estatura no mayor de los treinta centímetros, producción de semillas muy pequeñas, menor o igual a un milímetro, dos características que las hace poco atractiva para la fauna silvestre. Internacionalmente se recomienda que los aeropuerto deben de tener la menor diversidad de especies vegetales formando parte de sus áreas verdes (las que por ende son áreas manejadas), esto facilita el control de la vegetación e incluso el control de la fauna silvestre asociada (Cleary \& Dolbeer, 2005), como se verá en los resultados de esta investigación, específicamente en la parte de composición florística, existe una alta riqueza de especies tanto de gramíneas como de especies de hojas anchas formando parte de las áreas verdes del Aeropuerto Internacional Augusto C. Sandino.

Para el ensayo, se empleó la metodología recomendada por el CIAT (1981) para evaluación de especies herbáceas, en los llamados Ensayos Regionales de Adaptación (ERA). Se establecieron parcelas de $5 \times 5 \mathrm{~m}$ en las zonas donde se encuentran las mayores densidades de Panicum maximun. Dentro de las parcelas se evaluó la adaptación de dos especies de pastos seleccionadas para establecer en las áreas verdes del aeropuerto: Cynodon dactylon (Pasto Bermuda) y Botriochloa pertusa (Pasto Rodes). La selección de estas especies se hizo dentro del aeropuerto, ya que este material se encuentra dentro de las áreas verdes. Se establecieron nueve parcelas de $5 \mathrm{x} 5 \mathrm{~m}$, tres al este del aeropuerto, tres al sur y tres al oeste. Las parcelas fueron divididas en dos subparcelas de 2.5 x $5 \mathrm{~m}$ donde se ubicaron cada una de las especies evaluadas, se establecieron 5 hileras y 10 plantas por hileras, para un total de 50 plantas por subparcela.

El periodo del establecimiento para las dos especies fue de dos meses (Octubre a Noviembre), se procuro desarrollo de las gramíneas. Se utilizo un diseño completo al azar (DCA), donde se evaluó el comportamiento de cada una de las variables dentro de las especies y sitios. La observación del crecimiento del pasto se hizo cada 10 días después de su establecimiento donde se tomaron los datos de altura de las especies, números de entrenudos, ancho y largo de la hoja, porcentaje de establecimiento según lo observado.

Se realizó una prueba aposterioris de separación de media de Duncan o prueba de hipótesis de medias, esta prueba es útil para seleccionar él o los tratamientos y se aplican cuando el análisis de varianza declara diferencias significativas.

\section{RESULTADOS Y DISCUSIÓN}

Composición de especie. En total se registraron 11998 individuos, 11429 en el AVP, 485 en el BSO y 84 en la FA. En cuanto al estrato arbóreo, en las parcelas de $10 \times 10 \mathrm{~m}$ se contaron 117 individuos en los tres sitios, los cuales están agrupados en 11 especies, 10 géneros, ocho familias y cuatro órdenes. Las especies más abundantes según el número de individuos fueron: Albizia niopoides (27), Calycophyllum candidissimum (27), Azadirachta indica (18) y Senna siamea (16), el resto esta representado por menos de 10 individuos. Las familias más representadas según el número de especies fueron Caesalpiniaceae (2), Boraginaceae (2) y Anacardiaceae (2). Los órdenes más representados según el número de familias fueron: Fabales (3) y Sapindales (2). Para el estrato arbustivo, en las parcelas de 5 x $5 \mathrm{~m}$ se contaron 36 individuos, uno de Capsicum annum y 35 de Lantana camara en la FA, BSO y en el AVP. En las parcelas de 2 x $2 \mathrm{~m}$ se contaron 11845 individuos del estrato herbáceo en los tres sitios, agrupados en 28 especies, incluyendo una desconocida de la familia Malvaceae y cuatro identificadas hasta género (Desmodium sp, Asclepia $s p$, Sthenothaprum sp y Heliconia $s p$ ), contenidas en 23 géneros, 14 familias y 13 órdenes. Las especies herbáceas más abundantes fueron Cenchrus brownii (3 998 individuos), Cynodon dactylon (2 208), Digitaria decumbens (1 666), Bothriochloa pertusa (800), Elytaria imbricata (606) y Panicum maximum (534), el resto esta representado por menos de 500 individuos. Las familias más representadas fueron Poaceae (10) y Fabaceae (5). El orden más representado fue Lamiales (2).

Riqueza, densidades y diversidad de la vegetación comparada entre sitio. Se identificaron nueve especies arbóreas en la FA, seis en el BSO y dos en el AVP (Figura 2). Las mayores densidades de árboles se 
determinaron en la FA, debido a que históricamente, ese sitio ha sido reforestado con fines de crear sombra y de dar estética a las áreas verdes. Entre las especies con mayores densidades se encuentran: $C$. candidissimum (450 individuos/ha), A. niopoides (344), A. indica (289), S. siamea (261), el resto de las especies tenían densidades menores a los 133 individuos/ha. C. dentata y $J$. nervosa fueron las únicas especies de árboles que se determinaron en el AVP. Las densidades de árboles entre los tres sitios difieren significativamente $(\mathrm{KW}=11.53$, $\mathrm{p}<0.0069)$. Solamente se determinaron dos especies de arbustos: Lantana camara en AVP (2 320 individuos/ ha) y BSO (1200), y Capsicum annum en la FA (400).

De los tres sitios el AVP fue el que obtuvo los mayores registros de riqueza y densidad de herbáceas, principalmente por que en la historia, estas áreas eran destinadas como pastura para la ganadería y se presume que esos terrenos se usaron como sitios experimentales para establecer gramíneas para el ganado. Se determinaron 24 especies herbáceas en el AVP, ocho en el BSO y ninguna en la FA (Figura 2). Según el estimador Chao2 en el AVP restan determinar 6 especies más de plantas herbáceas, a medida que se incremente el esfuerzo de muestreo.

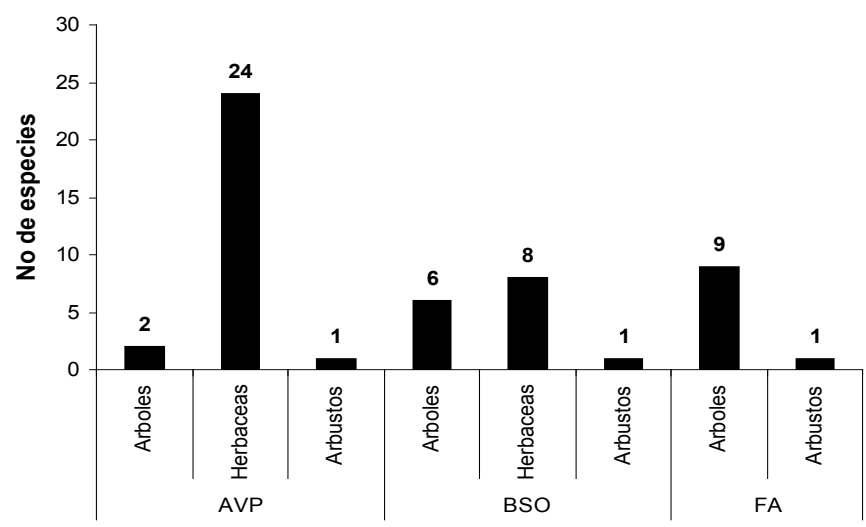

Figura 2. Riqueza de especies arbóreas, herbáceas y arbustivas distribuida en los tres sitios de muestreo.

Las especies con mayores densidades en el AVP fueron: Cenchrus brownii (256 282.05 individuos/ ha), $C$. dactylon (141 538.46), $D$. decumbens (106 794.87),B.pertusa (51282.05),Elytraria imbricada (38 846.15) y P. maximum (30 192.31), entre ellos las diferencias resultaron ser altamente significativas $\left(\mathrm{X}^{2}=30.84, \mathrm{p}<2.4 \mathrm{E}-9\right)$. Sumadas las densidades de estas cinco especies representan el $85.32 \%$ de la densidad de herbáceas total. Y en el BSO se fueron Cenchrus ciliaris (19 487.18) y Rhynchosia minima (17 307.69) sin diferencias significativas $\left(\mathrm{X}^{2}=1, \mathrm{p}>0.32\right)$.
La diversidad de árboles según el índice de Shannon-Wiener fue mayor en la FA (1.733), comparada con el BSO (1.145). En cuanto a hierbas la diversidad fue mayor en el AVP $(2,058)$ comparada con el BSO (1.719). En el caso los árboles del AVP y los arbustos del AVP y BSO no aplican el cálculo del índice porque solamente están representados por una especie.

Estructura vertical de la vegetación. Las especies arbóreas con mayor promedio de altura fueron: $S$. siamaea (11.75 m), S. mombin (10), C. fistula (10), A. indica (9.53), C. candidissimum (9.52) y A. niopoides (8.92), el resto de las especies poseen promedios de altura menores de $8 \mathrm{~m}$. Las diferencias de altura entre las especies resultó no ser significativa $\left(\mathrm{X}^{2}=2.077, \mathrm{p}>0.15\right)$. Los arbustos determinados tienen altura menores a un metro. Las especies herbáceas con mayores promedios de altura resultaron ser: $P$. maximum $(1.50 \mathrm{~m}), D$. tortuosum (1), Desmodium sp (0.77) y M. nivea (0.69), el resto de las especies tienen alturas menores a los $0.5 \mathrm{~m}$. Las diferencias de altura entre las especies resultaron ser significativas $\left(\mathrm{X}^{2}=13.62, \mathrm{p}<2.2 \mathrm{E}-4\right)$.

Fenología. De las 41 especies de árboles, arbustos y herbáceas identificadas en las áreas verdes del aeropuerto, se determinó el período de floración y fructificación de 31 de ellas, mediante observación de campo y revisión bibliográfica. De estas 6 (19.35\%) florecen y fructifican todo el año, entre estas: Cordia dentata, Boerhavia erecta, Chamaesyce hyssopifolia, Eleucine indica, Melanthera nivea y Rhynchosia minima. El resto florece y fructifica menos de nueve meses al año, y otros florece los doce meses del año, pero fructifican menos de cinco meses al año, entre estas: Cassia fistula y Lantana camara. Los meses de agosto-noviembre son los meses en que la mayoría de las especies $(65 \%)$ se encuentran floreciendo. De abril a agosto la mayor parte de las especies (del 52 al 61\%) esta produciendo frutos. De las 10 especies de árboles a los cuales se les logró determinar su fenología, el $100 \%$ de ellas florece y fructifica desde diciembre hasta septiembre, en el trimestre final del año solamente el $60 \%$ florece o fructifica.

En cuanto a las plantas herbáceas, de las especies a las cuales se les determinó su fenología completa, el $80.95 \%$ florece y fructifica en el período de diciembremarzo, $52.38 \%$ en el período marzo-junio, $95.24 \%$ en el período junio-septiembre y $76.19 \%$ en el período septiembre-diciembre. La presencia de frutos para las especies de herbáceas durante todo el año atrae las aves frugívoras y granívoras, si se sigue este tipo 
de vegetación continuara siendo difícil disminuir la presencia de la fauna durante todo el periodo. Todo lo anterior es evidencia que las especies nectarívoras, frugívoras e insectívoras (gremio alimenticio indirecto) tienen fuente de alimento todo el año.

Atractivos vegetales para la fauna silvestre. La constitución fisonómica y la diversidad de la vegetación de cualquier sitio determinan la presencia o ausencia de ciertas especies de fauna silvestre. Krebs (1985) expresa que la diversidad de estratos vegetales favorece la diversidad de aves, Emmons (1999) manifiesta que la diversidad de plantas favorece la diversidad de mamíferos frugívoros. La vegetación provee a la fauna silvestre de dos recursos fundamentales para la supervivencia y proliferación de cualquier especie silvestre: alimento y cobertura (Ojasti, 2000). Plantas con abundantes hojas (tupidas), siempre verdes, con flores con abundante néctar (suplen alimento a nectarivoros como Amazila rutila), frutos de tipo carnosos como bayas y drupas (suplen alimento a frugívoros como Aratinga canicularis), plantas que produzcan abundantes semillas o granos (suplen alimento a granívoros como al grupo de los Columbidos) y plantas o sitios que conserven y sean hábitat de insectos u otros invertebrados (brindan alimento a insectívoros como Crotophaga sulcirostris) son por lo general clasificadas como especies que atraen fauna aviar (Maldonado, Chalukian y Avedillo, 1999). De hecho otros autores como Cleary \& Dolbeer (2005), Hygnstrom, Timm \& Larson (1994), Godinez (2001) entre otros, manifiestan que los factores que atraen fauna silvestre a los aeropuertos son el alimento, el agua y el refugio (descanso y nidificación).

\section{Especies vegetales atractivas por brindar alimento} a la fauna silvestre. Una especie arbórea identificada como potencial en la producción de frutos fue Manguifera indica, el cual no fue cuantificando dentro de las parcelas de muestreo, pero se encuentra en abundancia muy cerca de los edificios y jardines dentro de los hangares, parqueos y áreas próximas a la pista, S. monbin produce frutos los cuales proveen de una exquisita fuente de alimento para especies de aves frugívoras como $A$. canicularis, Melanerpes hoffmani, Icterus pustulatus, entre otros, los cuales a su vez pueden ser presas de aves rapaces. Se determinaron especies de plantas potenciales en la producción de néctares como lo son Tabebuia rosea (no cuantificado dentro de las parcelas de muestreo), $A$. niopoides, C. cancicissimum, C. fistula, C. alliodora, $C$. dentata, G. ulmifolia y S. siamea (Salas, 1993). Todas como a $A$. rutila, la cual no presenta peligro para la aviación por su pequeño peso, hábitos solitarios, y por lo general es visitante de lugares con bastante cobertura vegetal. Especies de plantas herbáceas también son potenciales en la producción de flores pero a diferencias de los árboles y arbustos son menos visitadas por aves nectarívoras, en este caso, la fuente de alimento para las aves (lo que igualmente sucede con las flores de los árboles y arbustos) son los insectos que merodean las flores en busca de néctar o de refugio. La mayoría de las especies de aves tanto dentro como en los alrededores del aeropuerto se alimentan de invertebrados (entre ellos los insectos) y de granos (Figura 3), especies de aves como Thraupis episcopus, Campylorhynchus rufinucha, Dendroica petechia, Tyrannus melancholicus se dedican a forrajera por insectos entre las ramas de árboles y arbustos, otras especies como H. rustica, E. tula, Q. mexicanus, M. aeneus, E. superciliosa y Passer domesticus prefieren buscar insectos entre la vegetación herbácea, $H$. rustica en especial vuela y caza su presa en vuelo, lo cual la hace vulnerable a impactarse con un avión, al volar deliberadamente en el afán de perseguir en vuelo a sus presas.

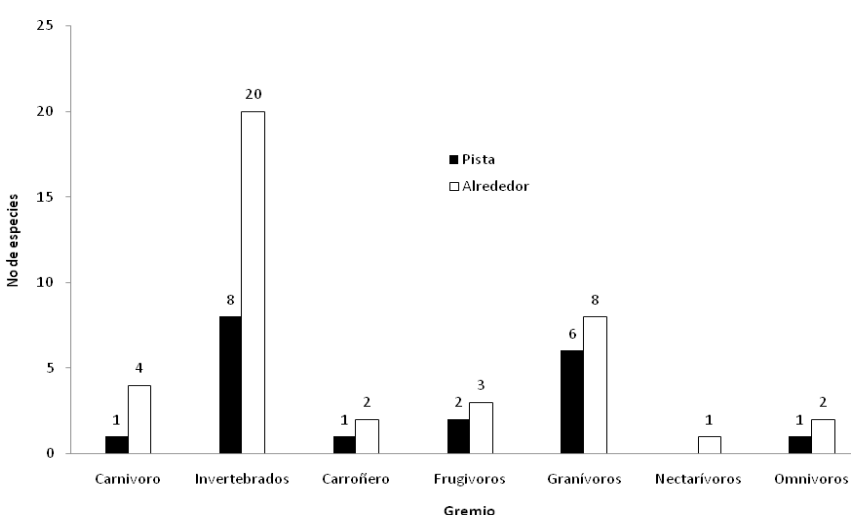

Figura 3. Número de especies por gremios alimenticios para las aves identificadsa alrededor de la pista de aterrizaje (Pista) y alrededor del aeropuerto (Alrededor).

En las áreas verde alrededor de la pista de aterrizaje, la mayoría de estos insectos (adultos, juveniles y larvales) apetecidos por las aves pertenecen a los órdenes Lepidopteras, Ortopteras y Coleópteras, estos se encuentran restringidos a las flores, en la parte inferior de las herbáceas (principalmente de las gramíneas) o dentro de los pastos secos que yacen en el suelo después de la chapoda. Otros invertebrados apetecidos por las aves y mamíferos medianos en ésta áreas son los Oligoquetos (principalmente en el período lluvioso) y Arácnidos. Un acontecimiento importante es la chapoda, ya que al chapodar y controlar el pasto en las áreas verdes 
alrededor de la pista, los insectos son perturbados y vuelan o quedan expuestos a las aves, de tal forma que especies como $H$. rustica (principalmente), E. tula, $M$. aeneus y $Q$. mexicanus siguen al vehículo que realiza la chapoda para aprovechar esa situación y obtener una provisión de insectos de una manera fácil, en este sentido la poda puede promover la aglomeración de aves que podrían entorpecer las operaciones aeroportuarias.

Por otra parte la chapodadora al momento de desarrollar su trabajo, mata mamíferos pequeños y medianos, reptiles y anfibios que ocupan los pastos altos como $P$. maximun, $P$. antidotale, Sorgum halapensis y $A$. gayanus (los tres últimos no se determinaron dentro de las parcelas de muestreo que se establecieron aleatoriamente) para construir sus madrigueras o para utilizarlos como corredor y desplazarse de un lugar a otro bajo la protección vegetal. Cuando estos animales son muertos se convierten en un atractivos para las aves de rapiña como para el peligroso C. atratus o los menos peligrosos C. aura y Polyborus plancus. Esos pequeños animales habitantes de los alrededores de la pista de aterrizaje también pueden ser fuente de atracción para aves rapaces diurnos como Buteos, Falcos o Accipiteres o para aves carnívoras nocturnas como Tito alba (Lechuza de los campanarios), Ciccabas, Otus, etc.).

En los meses de febrero-abril en que el período seco es marcado, hay una considerable reducción de especies floreciendo y fructificando, sin embargo ciertas especies de plantas herbáceas de hojas anchas alrededor de la pista de aterrizaje se mantienen floreciendo y fructificando aprovechando que la mayoría de los pastos están secos, representando una fuente de alimento para las aves y para los insectos que también sirven de alimento a estas aves, dentro de estas se pueden mencionar a Tridax procumbens, Tribulus terrestris, Tephrosia cinerea, Passiflora phohetida y algunas del género Desmodium. Se hace referencia especial a $P$. phohetida cuyos frutos en la mayoría de los casos fueron encontrados con orificios y sin semillas, rastro que evidencia a esta especie como potencial fuente de alimento para las aves, se presume que $Q$. mexicanus o algún Columbido era el responsable de eso. P. phohetida no se determinó en altas densidades dentro de las parcelas de muestreo, pero si se observo abundante en las áreas marginales de los caminos y pista de aterrizaje.

Los pastos son fuentes indiscutible de alimento, principalmente para especies de aves estrictamente granívoras como lo son Columbina talpacoti, Columba flavirostris, Columba livia, Zenaida asiatica, Colinus cristatus y a especies que complementan su alimento con granos como $P$. domesticus, $M$. aeneus y T. episcupus, de estas especies $C$. talpacoti, $C$. livia y $M$. aeneus resultaron con altos índices de peligrosidad (Muñoz y Martínez, 2009).

Especies vegetales atractivas por brindar cobertura (hábitat y refugio) a la fauna silvestre. La cantidad de especies y la abundancia de los árboles en la FA, la estructura vegetal formada por árboles dispersos de $A$. niopoides y el sotobosque dominado por $P$. maximun con alturas de hasta dos metros, y la diversidad vegetal y estructural de las plantas en la UN, US y SR proveen de refugios a vertebrados terrestres en los alrededores y dentro del aeropuerto.

Por otra parte la altura y el tipo de pasto le proporciona excelente condiciones de hábitat y alimento a mamíferos pequeños y medianos principalmente Roedores, Sylvilagus sp (Conejos), Conepatus semistriatus (Zorrillos), algunos reptiles como Ctenosaura similis (Garrobo negro), Conophis lineatus (Culebra lagartijera), Mabuya unimarginata (Lagartija rayada) y anfibios como Bufo marinus (Sapo común), todos estos animales mueren en el momento de la chapoda y atraen con su muerte a miembros de la especie $C$. atratus, según reporte del personal de mantenimiento. Especies de mamíferos silvestres de tamaño grande son poco probables que se presenten en las áreas dentro del aeropuerto, debido a la restricción del acceso que representa el enmallado perimetral, aunque no se descarta la posibilidad de que perros callejeros puedan ingresar al aeropuerto entre los causes.

Especies como Nyctidromus alvicollis, C. cristatus anidan en el suelo y para ellos la cobertura de pastos altos es necesaria. Z. asiatica, C. talpacoti y Sturnella magna ocasionalmente se encuentran forrajeando bajo la protección de la vegetación. Muñoz y Martínez (2009) reportan especies arbóreas potenciales para la anidación según el conteo de nidos en el arbolado dentro y próximo a la infraestructura están Terminalia catapa (donde se encontraron mayor cantidad de nidos), Acacia sp, Manguifera indica (segundo con mayores albergues de nidos), Citrus sp, Cocos nucifera y Tecota stans.

El manejo de las cortas o chapodas son de vital importancia para el abastecimiento de material orgánico para todos las plantas que se encuentran en el AVP y el BSO, ya que el material cortado no se extrae y se transforma en humus revertiendo los nutrientes al suelo para que sean utilizados por las nuevas plantas o por los retoños, formando un "Ciclaje de nutrientes" en el que las plantas extraen nutrientes un tanto profundo en la tierra para formar parte de su biomasa y luego 
al ser cortadas y dejadas sobre la superficie del suelo, éstas se descomponen y los nutrientes extraídos quedan disponibles para formar un ambiente rico a semillas y retoños. De hecho los suelos del aeropuerto son ligeramente ácidos y medianos en materia orgánica, esto último principalmente donde hay pastos altos.

Por otra parte el pasto seco tirado sobre las áreas verdes son ideales para que mamíferos pequeños $\mathrm{y}$ medianos y serpientes hagan sus madrigueras, en campo se observo madrigueras por debajo del material seco producto de la chapoda que formaban pasadizos conectados, con rastros de desplazamiento de algún animal de tamaño mediano. El pasto seco es utilizado potencialmente en la elaboración de los nidos de las especies de aves que residen dentro del aeropuerto.

No se encontró ningún registro bibliográfico ni en las observaciones en las áreas de estudio que determinaran el potencial atractivo para la fauna silvestre de las dos especies arbustivas encontradas dentro de las unidades de muestreo, pero se presume que las flores de L. camara por sus colores pueden ser potenciales en la atracción de insectos que a la vez pueden atraer aves insectívoras.

Pero no todas las especies son tan atractivas especies como Cenchrus brownii y C. ciliaris (la especie con mayor densidad en el AVP) presentan frutos recubiertos por cipselas erizadas espinosas que las hace inatractivas para las aves. Especies de gramíneas de tamaño pequeño (menos de $30 \mathrm{~cm}$ ) como C. brownii, C. dactylon, B. pertusa, Paspalum notatum, etc. promueven que el desplazamiento de vertebrados no aves sea reducida, ya que son pocos los animales que toleran áreas donde están muy visibles o expuestos a depredadores o al ruido de la actividad aeroportuaria. Afortunadamente el área alrededor de la pista de aterrizaje esta dominada por $C$. brownii reduciendo la posibilidad de que ese sitio sea de refugio para mamíferos pequeños o reptiles, siendo de condición opuesta las áreas próximas al enmallado perimetral del aeropuerto (la mayor parte del área alrededor de la pista) donde hay dominancia de $P$. maximum (Figura 4).

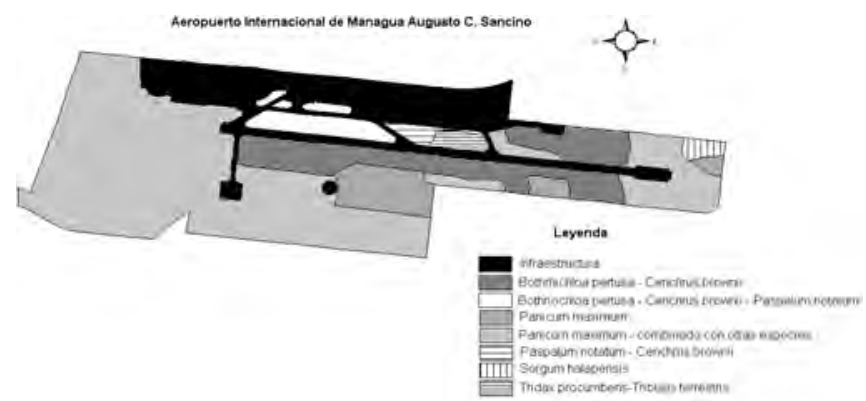

Figura 4. Estratificación vegetal basada en las especies de mayor dominancia.
Adaptación y establecimiento de especies vegetales poco atractivas para la fauna silvestre. Con el fin de recomendar especies de pastos que sean poco atractivos directa o indirectamente para los pájaros (principalmente para las especies de aves peligrosos para la aviación) que se puedan establecer en las áreas verdes del aeropuerto, se seleccionaron dos especies Cynodon dactylon (Pasto Bermuda) y Botriochloa pertusa (Pasto Rodes), evaluándose en ellas su adaptación y establecimiento en dichas áreas. Dentro de los criterios que se usaron para seleccionar dichas especies están: ser potenciales para su establecimiento, se encuentran su baja estatura no mayor de los treinta centímetros, sin semillas o con semillas muy pequeñas, menor o igual a un milímetro, dos características que las hace poco atractiva para la fauna silvestre y finalmente es necesario que las plantas tengan mucha agresividad en la colonización, factores que se encontraron en ambas especies.

Las dos especies se lograron establecer satisfactoriamente, pero $C$. dactylon presentó los incrementos significativos en cuanto al desarrollo en altura, largo de la hoja y número de nudos en comparación con $B$. pertusa.

\section{CONCLUSIONES}

En total se contaron 11998 individuos vegetales dentro de las unidades de muestreo, de estos, 117 son árboles los cuales están agrupados en 11 especies, 10 genero, 8 familias y 4 ordenes, 36 son arbustos agrupados en dos especies, dos genero, dos familias y dos ordenes y 11845 individuos de especies herbáceas agrupados en 28 especies incluyendo una desconocida, contenida en 23 géneros, 14 familias y 13 ordenes.

La mayor riqueza, densidad y diversidad de arboles y herbáceas se identificaron en la FA y el AVP respectivamente.

La vegetación resulta un foco de atracción para especies de aves peligrosas para la aviación de una forma directo o indirecta al brindarle alimento y refugio a la fauna silvestre.

A nivel de árboles, las especies identificadas como atractivas (directos e indirectos) por brindar refugio, alimento y hábitat resultaron ser: Spondias mombin, Manguifera indica, Cordia dentata, Cordia alliodora, Cassia fistula, Senna siamea, Azadirachta indica, Callycophyllum candidisimun, Guazuma ulmifolia y Senna siamea.

En cuanto a herbáceas, las especies identificadas como atractivas (directos e indirectos) por brindar refugio, alimento y hábitat fueron: Chamaesyce hyssopifolia, Passiflora phohetida, Eleucine indica, 
Ixophorus unisetus, Panicum antidotale, Tridax procumbens y Tribulus terrestris.

De las 41 especies de árboles, arbustos y herbáceas, se determinó el período de floración y fructificación de 31 de ellas. De estas 6 (19.35\%) florecen y fructifican todo el año, entre estas: Cordia dentata, Boerhavia erecta, Chamaesyce hyssopifolia, Eleucine indica, Melanthera nivea y Rhynchosia minima.
Se concluye que dos de las especies potenciales para ser establecidas en las aéreas verdes del aeropuerto resultaron ser: Cynodon dactilon y Bothriochloa pertusa y de estas dos la especie $C$. dactilon es la que mejor creció y se estableció, principalmente en los sectores centro medio, este medio, este sur, centro norte y oeste norte.

\section{REFERENCIAS BIBLIOGRÁFICAS}

Cleary, E. \& Dolbeer, R. 2005. Manejo del riesgo por fauna silvestre en aeropuertos. Manual para personal aeroportuario, segunda edición marzo del 2005. Washintong Estados Unidos 384p.

Colwell, R. K. 2004. EstimateS, Statistical Estimation of Species Richness and Shared Species from Samples. (programa de cómputo) Versión 7.00. University of Connecticut. United States.

CIAT CENTRO INTERNACIONAL DE AGRICULTURA TROPICAL, 1981. Manual para la Evaluación Agronómica., Red Internacional de Evaluación de Pastos Tropicales. Editor técnico: José M. Toledo. Cali, Colombia. 170p.

Emmons, L. H. 1999. Neotropical Rainforest Mammals. Editions sig. University of Chicago press, ISBN. 307p.

EAAI EMPRESA ADMINISTRADORA DE AEROPUERTOS INTERNACIONALES, 2006. Aeropuerto Internacional de Managua, Historia - Antecedentes; Creación de la Empresa Administradora de Aeropuertos Internacionales (EAAI) Ampliación Remodelación y Reforzamiento Estructural de la Terminal Internacional de Pasajeros del Aeropuerto Internacional de Managua - Facilidades. Empresa Administradora de Aeropuertos Internacionales (EAAI). Managua, NI. 27p.

Godinez, E. 2001. Programa Nacional de Limitación de la Fauna Silvestre en Aeropuertos de Panamá. Organización de Aviación Civil Internacional. 15p.

GRAPHPAD SOFTWARE, INC., 2000. GraphPad InStat. (programa de computo) Version 3.05.

Hammer, Ø. \& Harper, D. A. T. 2004. PAST PAlaeontological Statistics. (programa de cómputo) Versión 1.29. University of Oslo, Noruega.

Hygnstrom S., Timm R. \& Larson G. 1994. Prevention and Control of Wildlife Damage. US Department of Agriculture-Animal and Plant Health Inspection Service-Animal Damage Control and through the cooperation of the Natural Resources and Rural Development Unit, extension Service, US Department of Agriculture, Washington, DC, United States. 840 p.

Krebs, 1985. Ecología de la distribución y la abundancia. 2da edición 753p.

Maldonado, C., Chalukian, S. y Avedillo, M. 1999. Disfrute de las aves en su jardín. Escuela Agrícola Panamericana, El Zamorano, Honduras, Centro América. 41p.

Moreno, C. E. 2001. Métodos para medir biodiversidad. Centro de investigación, Universidad Autónoma del estado de Hidalgo. Edición CYTED. Pachuca, Hidalgo, México.

Muños, P. A. y Martínez, A. 2009. Análisis de riesgo de impacto en el aeropuerto internacional Augusto c. Sandino Managua, Nicaragua.

Ojasti, J. 2000. Manejo de Fauna Silvestre Neotropical. SI/ Monitoring \& Assessment of Biodiversity Program (SI/ MAB). Instituto de Zoología Tropical, Universidad Central de Venezuela, Caracas, Venezuela. 290p.

Salas, J.B. 1993. Árboles de Nicaragua. Instituto Nicaraguense de Recursos Naturales y del Ambiente, IRENA. Managua, Nicaragua.390p.

Stevens, W. Ulloa, C. Pool, A \& Montiel, O. e.d.2001. Flora de Nicaragua tomo I, II y III. Missouri Botanical Garden. U.S.A. 2556p. 